\title{
Modelo de redes neuronales para predecir la tendencia de víctimas de secuestro en Colombia
}

INVESTIGACIÓN E INNOVACION en INGENERÍAS ISSN2344-8652

Santiago Giraldo Alegría

\section{Neural network model for predicting kidnapping victims trends in Colombia}

\begin{abstract}
OPEN ACACES
Recibido: 22/09/2020

Aceptado: 22/10/2020

Publicado: $23 / 11 / 2020$

Correspondencia de autores: giraldo.santiago@correounivalle. edu.co

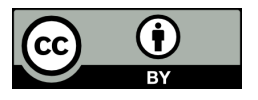

Copyrith 2020 by Investigación e Innovación en Ingenierías

$$
\text { de }
$$$$
\text { regis }
$$

r

\section{Luis Eduardo Ordoñez Palacios \\ Victor Bucheli Guerrero} Universidad del Valle, Colombia
Hugo Ordoñez Erazo

Universidad del Cauca, Colombia

Resumen

Objetivo: Entrenar Redes Neuronales y Máquinas de Soporte Vectorial para la predicción de tendencias futuras en el número de víctimas de secuestros por año en Colombia, con el fin de apoyar la toma de decisiones. Metodología: Se procesó un conjunto de datos de víctimas de secuestro con 39.863 registros tomado del portal de datos abiertos del gobierno y publicado por la Fiscalía Nacional de Colombia, con el fin de entrenar y evaluar una Red Neuronal y una Máquina de Soporte Vectorial para regresión (SVR), esto permitió identificar qué modelo tuvo menor Raíz del Error Cuadrático Medio (RMSE) para predecir la tendencia de víctimas de secuestro. Resultados: Se encontró que la Red Neuronal entrenada tuvo menor Raíz del Error Cuadrático Medio (RMSE) frente a la Máquina de soporte vectorial tanto para la predicción de víctimas de secuestro. Se pudo estimar la cantidad de víctimas de secuestros en los próximos diez años en todo el territorio colombiano. Conclusiones: Existen diversos trabajos relacionados con la predicción de delitos, sin embargo, este estudio propone un nuevo modelo de Redes Neuronales para estimar la tendencia de víctimas de secuestro en Colombia.

Palabras clave: Modelo de predicción, aprendizaje automático, Redes neuronales, crimen, secuestro. Abstract

Objective: Train Neural Networks and Support Vector Machines for regression for predicting future trends in kidnapping victims numbers by years in Colombia, with the purpose of support decision making. Methodology: A dataset about kidnapping victims with 39.863 records were processed, the dataset was taken from the government open data given by the Colombian Prosecutor's Office, with means of training and evaluating a Neural Network and a Support Vector Machine for regression (SVR), this allowed to identify the model with less Root Mean Squared Error (RMSE) for predicting kidnapping victims trends. Results: We found that the trained Neural Network had less Root Mean Squared Error (RMSE) against the Support Vector Machine and we were able to estimate the number of kidnapping victims in the next ten years throughout the Colombian territory. Conclusiones: There are several papers related to crime prediction, however, this study proposes a new model of Neural Networks to estimate the trend of kidnapping victims in Colombia.

Keywords: Prediction model, machine learning, neural networks, crime, kidnapping.

Como citar (IEEE): S. Giraldo-Alegría, S., L. Ordoñez-Palacios., V. Bucheli-Guerrero., y H. Ordoñez-Erazo. “ Modelo de redes neuronales para predecir la tendencia de víctimas de secuestro en Colombia", vol. 8, n³, pp. 38-49, 2020. DOI: https://doi. org/10.17081/invinno.8.3.4702 


\section{Introducción}

La delincuencia es un flagelo que conduce a la ejecución de actividades en contra de las leyes promulgadas por los gobiernos de cada país, la tipificación de los delitos es muy amplia y difiere en su denominación y contexto jurídico, sin embargo, la extorsión y el secuestro de civiles son reconocidos mundialmente como crímenes de lesa humanidad [1], aunque, en el presente, muchas naciones continúan registrando sucesos relacionados con este fenómeno, como se observa en [2].

Colombia es un país que ha sufrido las consecuencias del conflicto armado interno durante las últimas décadas [3]. De otra parte, el gobierno Colombiano ha incrementado gradualmente, el uso de nuevas tecnologías de la información y como muestra de ello, el Portal de Datos Abiertos permite que las entidades del sector público, publiquen información estructurada a disposición de los usuarios para generar estadísticas e investigaciones, entre otros.

El secuestro es un delito que según el Consejo Económico y Social de las Naciones Unidas [4]: "consiste en detener ilícitamente a una persona o personas en contra de su voluntad con la finalidad de exigir por su liberación un provecho ilícito o cualquier utilidad de tipo económico u otro beneficio de orden material, o a fin de obligar a alguien a que haga o deje de hacer algo"; en Colombia las cifras se han reducido en los últimos años; según la Revista de Criminalidad de la Policía Nacional de Colombia publicada en septiembre de 2019 [5], este delito registró una reducción del 11.28\% de los casos en 2018 comparado con el año 2017. Combatir el crimen del secuestro es fundamental para evitar la violencia, la degradación social y la descomposición de las familias [6], actualmente, se puede tomar ventaja de diversas tecnologías para apoyar el proceso de toma de decisiones, considerando la importancia de obtener los datos necesarios para cualquier estudio, la Fiscalía de Colombia en [7] ha dispuesto información que incluye datos como: año de ocurrencia del delito y de denuncia, departamento, municipio, número de víctimas, estado de la denuncia, etc.

Actualmente, las técnicas de inteligencia artificial permiten estudiar datos delictivos históricos, utilizando métodos estadísticos y de aprendizaje de máquina, obteniendo buenos resultados en el campo de la predicción, para anticipar el terrorismo y pronosticar conductas delictivas [8, 9]. A diferencia de los trabajos relacionados, esta investigación implementó, configuró y entrenó una Red Neuronal mediante Keras de Tensorflow y MLPRegressor de sklearn, con 6 capas ocultas, 505 nodos, una iteración máxima de 150 épocas y el algoritmo de optimización Adam [10]. Los resultados obtenidos permiten estimar el número de víctimas de secuestro por año.

El documento está compuesto por las siguientes secciones, así: Contexto del problema, trabajos relacionados, metodología, resultados y evaluación, y finalmente, las conclusiones y trabajo futuro.

\section{Contexto del problema}

Según [3], el conflicto armado colombiano es el más longevo de la región, se ha producido durante cinco décadas y está basado en controversias político-ideológicas, enfrentando al Estado colombiano, representado por sus Fuerzas Armadas y de Policía, con organizaciones guerrilleras y paramilitares financiadas por operaciones de narcotráfico y la práctica de delitos como el secuestro, la extorsión, entre otros. En la Figura 1 se puede apreciar el total histórico de víctimas de secuestro por cada departamento y en la Figura 2 se presenta el riesgo por presencia de Grupos Armados Organizados, tomados de [11]. 
Figura 1. Total Histórico de Víctimas en Colombia por Departamento

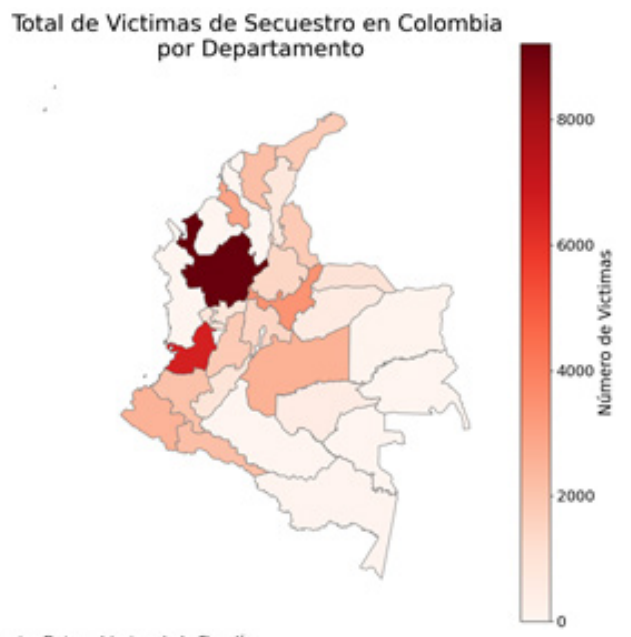

Fuente: Datos abiertos de la fiscalia

Fuente: Elaboración Propia

Figura 2. Riesgo por presencia de Grupos Armados Organizados
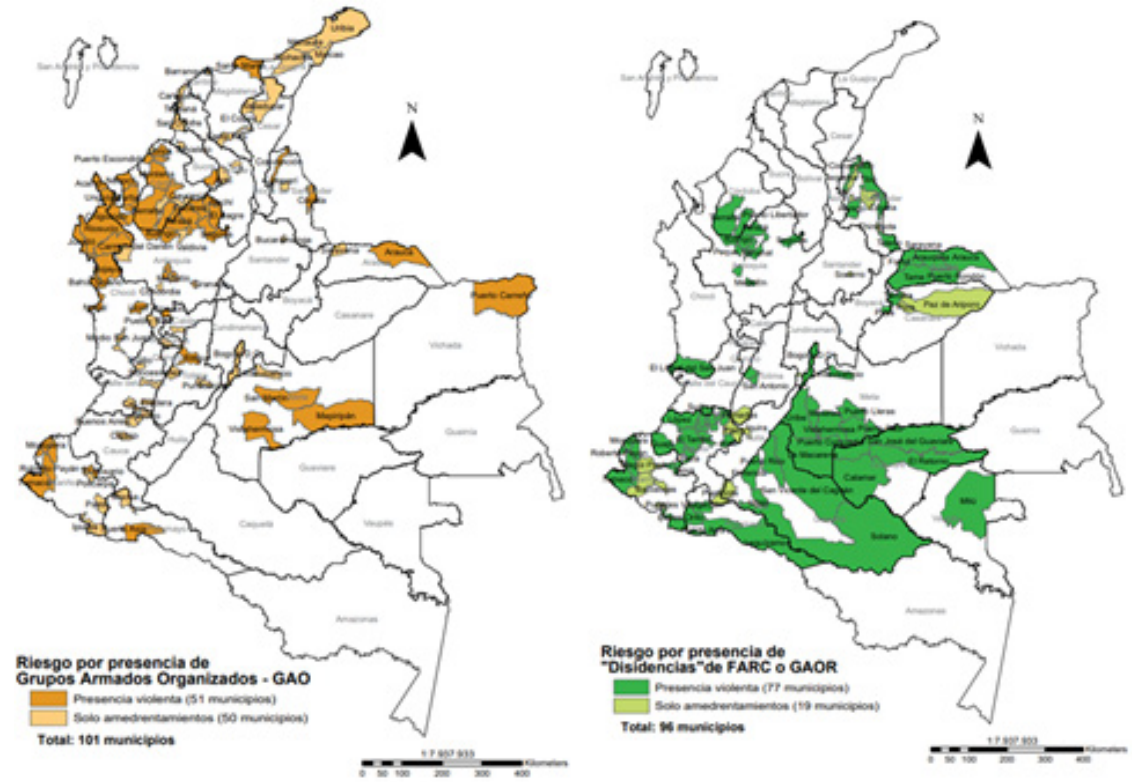

Fuente: Mapas y Factores de riesgo electoral. Elecciones de autoridades locales Colombia 2019 [11]

El secuestro es un delito que generalmente, es efectuado por grupos ilegales que toman parte en una confrontación armada con fines de financiamiento o de represalias, conforme a [5], al finalizar el año 2018, los departamentos de Norte de Santander (23), Cauca (22), Valle del Cauca (15) y Antioquia (14) presentaron los indicadores más altos. En la Tabla 1 se puede observar los diez departamentos con más víctimas registradas entre 1982 y 2020. 
Tabla 1. Top 10 Departamentos con más víctimas registradas históricamente

\begin{tabular}{|c|c|}
\hline Departamento & Total Victimas de Secuestro \\
\hline Antioquia & 9212 \\
\hline Valle del Cauca & 6621 \\
\hline Bogotá, D.C. & 5985 \\
\hline Caquetá & 3127 \\
\hline Sucre & 2925 \\
\hline Meta & 2590 \\
\hline Nariño & 2534 \\
\hline Magdalena & 2195 \\
\hline Putumayo & 2195 \\
\hline Cauca & 2107 \\
\hline
\end{tabular}

Fuente: Conteo de Víctimas - Datos Abiertos Colombia [11]

\section{Trabajos relacionados}

Las Redes Neuronales se emplean ampliamente en la predicción de eventos futuros en muchos campos de aplicación, algunas investigaciones se han enfocado en pronosticar tendencias de rendimiento de maquinaria rotativa en el sector industrial [12], de degradación de dispositivos en los sectores de energía y transporte [13], del mercado de valores en el sistema financiero [14], de emisiones antropogénicas en el sector ambiental [15] y de tendencias de influenza en el área de la salud [16].

En cuanto a la investigación judicial, se encontraron modelos de predicción de delitos asociados al secuestro, la extorsión, el homicidio, el hurto, entre otros, para apoyar a las entidades judiciales en el diseño de estrategias proactivas que permitan contrarrestarlo. Algunos trabajos emplean técnicas de Aprendizaje Automático por transferencia [17], supervisado y no supervisado como: Máquinas de Soporte Vectorial [9], Random Forest y Naïve Bayes [18], K-Nearest Neighbour [19], Clustering [20]; Árboles de Decisión [21], Métodos de ensamble [22] y Clasificadores híbridos [8]; del mismo modo, otros estudios utilizan algoritmos de Minería de Datos como las Reglas de Asociación [23] y Modelos Estadísticos según se observa en [24].

Los investigadores en [21], desarrollaron un modelo de Aprendizaje supervisado que utiliza Árboles de Decisión y el algoritmo ID3 para predecir las actividades criminales relacionadas con el secuestro de mujeres en la India. Del mismo modo, el trabajo de [18], recopiló registros de secuestro y otros delitos para analizar la tendencia del crimen en la India, además, realiza un estudio comparativo entre diferentes técnicas de clasificación, encontrando que los algoritmos Random Forest y Naïve Bayes proporcionan la mayor precisión en la predicción del secuestro. Ambos estudios evalúan el desempeño de los algoritmos mediante las métricas de la matriz de confusión y el área bajo la curva.

En la revisión, también se encontró que el Aprendizaje Profundo se ha utilizado para estimar la ocurrencia de los delitos en todas sus modalidades, según [25], estos algoritmos proporcionan mejores resultados de predicción comparados con otros métodos de pronóstico relacionados con el Aprendizaje Automático; por su parte, [26] propone nuevos modelos de Aprendizaje Profundo para el mapeo predictivo del crimen basados en características espacio-temporales.

El trabajo en [27], utilizó métodos de agrupamiento espacial y modelos de Redes Neuronales Artificiales para pronosticar zonas de alto riesgo de incidentes delictivos; la investigación recolectó 10778 datos del Departamento de la Policía de Chicago, los delitos estaban relacionados con el secuestro, homicidios, 
hurtos, entre otros; en el estudio se probaron diferentes tecnologías y se encontró que el modelo óptimo tenía una topología de 24 neuronas en la capa oculta y en consecuencia, el error cuadrático medio (MSE), fue de 0,00013833 .

La investigación en [28], empleó Redes Neuronales para predecir la reincidencia criminal como una alternativa a los modelos estadísticos tradicionales, los datos se obtuvieron del Consorcio Interuniversitario de Investigación Política y Social de la Universidad de Michigan y se analizaron con la técnica de Red Neuronal prealimentada usando nueve nodos de entrada correspondientes a las variables explicativas, los resultados mostraron mayor desempeño cuando la configuración de la capa oculta tenía 39 nodos con un $69.20 \%$ de clasificaciones correctas, aunque la red con 26 nodos funcionó casi tan bien con un $69.17 \%$.

\section{Metodología}

En este apartado, se determinan las preguntas de interés que llevaron a la construcción de un modelo para predecir tendencias de secuestro en Colombia utilizando Redes Neuronales y comparando los resultados con Máquinas de Soporte Vectorial para regresión (SVR por sus siglas en inglés), también se describe la fuente de información y la manera en cómo se procesaron los datos. Esta investigación se produce para responder a: ¿La cantidad de datos es suficiente para predecir tendencias de secuestro?, ¿Una Red Neuronal es la mejor técnica para predecir tendencias de datos de secuestro en Colombia?, y ¿Cuál es la precisión de las estimaciones realizadas?, estos interrogantes se resuelven en el transcurso del documento.

El modelo utiliza un conjunto de datos tomado del portal de datos abiertos del gobierno y publicado por la Fiscalía Nacional de Colombia, el cual, cuenta con un total de 39.863 registros de secuestros realizados en Colombia entre los años 1982 y 2020, donde se especifica el total de víctimas en cada caso.

Del conjunto de datos original se descartaron datos nulos, incompletos, y que no tuvieran referencia a colombia, ya que hay registros de secuestros en otros países.

Para entrenar los modelos se agrupó el total de víctimas por año en que ocurrió el secuestro y se procedió a generar un dataset de 6 columnas, donde las primeras 5 columnas corresponden a una ventana de tiempo de 5 años y la última columna corresponde al año siguiente.

La Red Neuronal se implementó usando Keras de Tensorflow y MLPRegressor de sklearn, así mismo, se configuró con 6 capas ocultas y 505 nodos y fue entrenada con una iteración máxima de 150 épocas y el algoritmo de optimización Adam [10]. Los datos de entrada de la red corresponden en un porcentaje del $85 \%$ a datos de entrenamiento, y un porcentaje del $15 \%$ a datos de prueba para evaluar el modelo; la división de datos de entrenamiento y prueba se realizó sin modificar el orden temporal de los datos. Considerando que los pesos en la Red Neuronal se establecen de forma aleatoria al iniciar el entrenamiento, las implementaciones se entrenaron y almacenaron en varias oportunidades hasta hallar los resultados con menor error cuadrático medio. En la Figura 3, se aprecia la Red Neuronal compuesta por capas densas.

La Máquina de Soporte Vectorial para regresión (SVR) se implementó con un kernel lineal y los parámetros C con un valor de 1.0, epsilon con un valor de 0.1 y una tolerancia de 1×10-3. 
En la Figura 4 se observa el procedimiento aplicado al dataset en cada una de las etapas del modelo, el preprocesamiento de los datos y los pasos seguidos por la Red Neuronal y la Máquina de Soporte Vectorial desde la fuente de información hasta las predicciones de tendencias de secuestro en Colombia.

Figura 3. Estructura propuesta de Red Neuronal

\begin{tabular}{|c|c|}
\hline \multicolumn{2}{|c|}{ Capa de Entrada } \\
\hline Entrada & Salida \\
\hline$(?, 5)$ & $(?, 5)$ \\
\hline
\end{tabular}$\rightarrow$\begin{tabular}{|c|c|c|c|}
\hline \multicolumn{2}{|c|}{ Capa Oculta Densa } \\
\hline Entrada & Salida \\
\hline$(?, 5)$ & $(?, 5)$ \\
\hline
\end{tabular}$\quad$\begin{tabular}{|c|c|c|}
\hline \multicolumn{2}{|c|}{ Capa Oculta Densa } \\
Entrada & Salida \\
\hline$(?, 5)$ & $(?, 200)$ \\
\hline
\end{tabular}$\quad$\begin{tabular}{|c|c|c|}
\multicolumn{2}{|c|}{ Capa Oculta Densa } \\
Entrada & Salida \\
\hline$(?, 200)$ & $(?, 150)$ \\
\hline
\end{tabular}

\begin{tabular}{|c|c|c|c|c|c|}
\hline \multicolumn{2}{|c|}{ Capa Oculta Densa } & \multicolumn{2}{|c|}{ Capa Oculta Densa } & \multicolumn{2}{|c|}{ Capa de Salida } \\
\hline Entrada & Salida & Entrad & Salida & Entrada & Salida \\
\hline$(?, 150)$ & $(?, 100)$ & $(?, 100$ & $(?, 50)$ & $(?, 50)$ & $(?, 1)$ \\
\hline
\end{tabular}

Fuente: Elaboración propia

Figura 4. Flujo de información del modelo

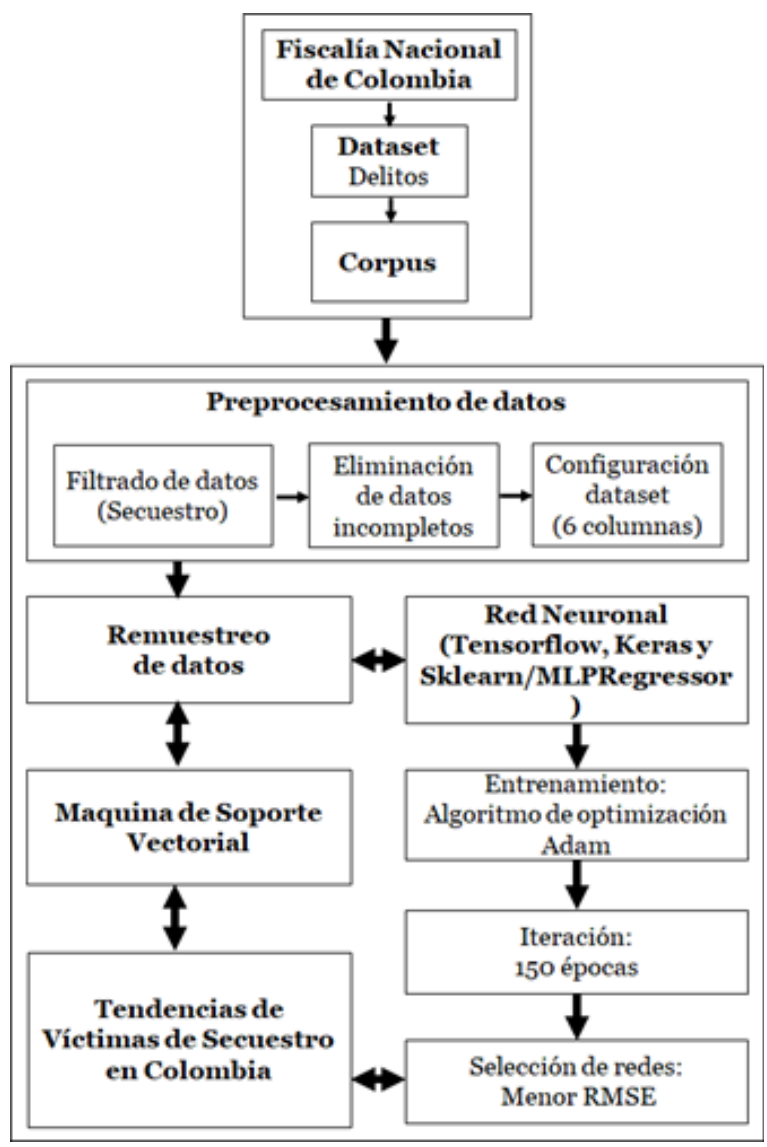

Fuente: Elaboración propia 


\section{Resultados}

Según la organización del dataset descrito en la metodología donde las primeras 5 columnas corresponden a un periodo de tiempo de 5 años y la última columna corresponde al año siguiente, se obtuvo un nuevo dataset que modela la tendencia desde 1982 hasta 2020 y se entrenaron los modelos para predecir la tendencia hasta 10 años en el futuro. Como ejemplo, la Tabla 2 presenta la cantidad de datos de entrada y salida de los primeros años entre 1982 y 1990.

Tabla 2. Primeros años: 1982 a 1990

\begin{tabular}{|c|c|c|c|c|c|}
\hline 1982 & 1983 & 1984 & 1985 & 1986 & Salida \\
\hline 24 & 1 & 1 & 3 & 11 & 45 \\
\hline 1983 & 1984 & 1985 & 1986 & 1987 & Salida \\
\hline 1 & 1 & 3 & 11 & 45 & 46 \\
\hline 1984 & 1985 & 1986 & 1987 & 1988 & Salida \\
\hline 1 & 3 & 11 & 45 & 46 & 42 \\
\hline 1985 & 1986 & 1987 & 1988 & 1989 & Salida \\
\hline 3 & 11 & 45 & 46 & 42 & 358 \\
\hline
\end{tabular}

Fuente: Elaboración propia

Los valores de la raíz del error cuadrático medio (RMSE por sus siglas en inglés) para cada modelo se pueden observar en la Tabla 3.

Tabla 3. Valores del RMSE para los modelos evaluados

\begin{tabular}{|c|c|c|}
\hline Modelo & RMSE Entrenamiento & RMSE Prueba \\
\hline Máquina de Soporte Vectorial (SVR) & 608.98 & 686.40 \\
\hline Red Neuronal MLPRegressor & 180.78 & 593.42 \\
\hline Red Neuronal Keras & 148.60 & 555.03 \\
\hline
\end{tabular}

Fuente: Elaboración propia

La Figura 5 presenta los resultados obtenidos del entrenamiento de los modelos. La primera gráfica son los datos de víctimas por año desde el año 1982 al año 2020, y las gráficas siguientes son la predicción de cada una de las implementaciones para los datos de entrenamiento y de prueba, con estas gráficas se busca comparar qué tanto se ajustan los modelos entrenados a los datos originales. Cada gráfica tiene una línea de regresión lineal simple que no es suficiente para representar la tendencia de los datos.

Aunque la Máquina de Soporte Vectorial se adaptó a los valores de entrenamiento y prueba resultando en valores de RMSE iguales a 608.98 y 686.40 respectivamente, la Red Neuronal logró adaptarse mejor y modelar la tendencia del descenso de víctimas en los últimos años lo cual se observa en las Figuras 6, 7 y 8 donde la predicción de la Máquina de Soporte Vectorial se queda estancada. 
Figura 5. Resultados obtenidos en entrenamiento y pruebas
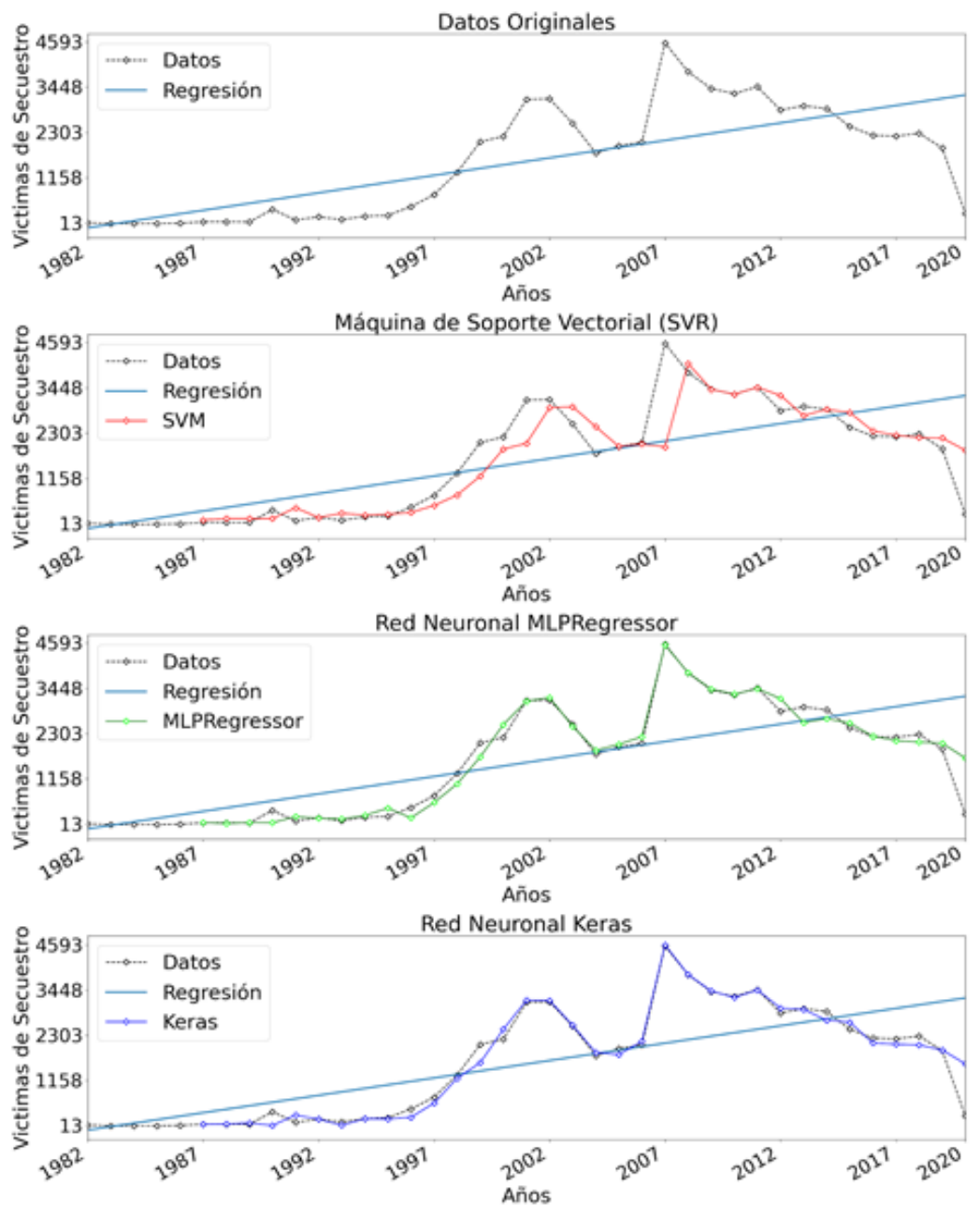

Fuente: Elaboración propia

Las Figuras 6, 7 y 8 muestran la predicción de víctimas de secuestro desde el año 2020 hasta el año 2030 de acuerdo a cada uno de los modelos. Se toma como punto de partida los años 2015 al 2019 para predecir los años siguientes, de igual manera se toma desde el año 2016 al valor predicho para 2020 para predecir 2021 y así sucesivamente, se excluye 2020 de los datos iniciales debido a que el procesamiento de los datos de este documento ocurre a la mitad del año 2020 y pueden generarse más registros al finalizar el año.

Se puede observar que los resultados de la Máquina de Soporte Vectorial no se ajustan a la tendencia y muestran cierto estancamiento, mientras que la Red Neuronal valida el comportamiento del descenso de víctimas para los últimos años en ambas implementaciones y se ve justificado por los valores del RMSE. 
Figura 6. Predicción de víctimas de la Máquina de Soporte Vectorial al año 2030

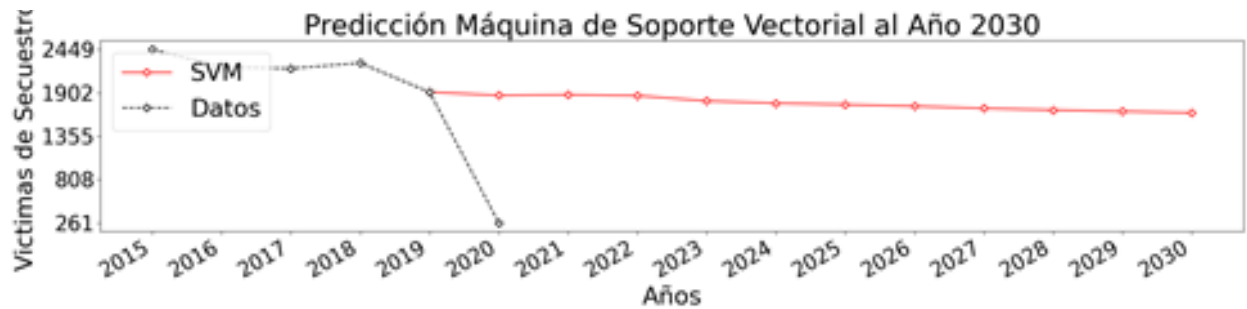

Fuente: Elaboración Propia

Figura 7. Predicción de víctimas de la Red Neuronal al año 2030 (MLPRegressor)

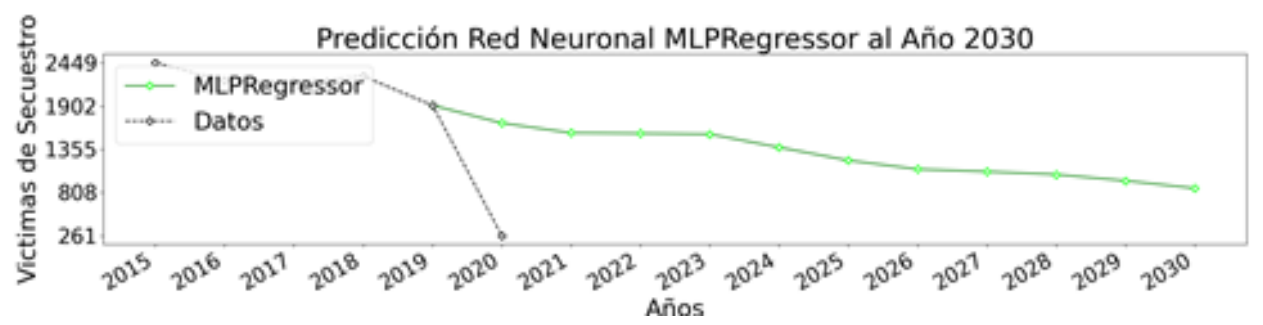

Fuente: Elaboración propia

Figura 8. Predicción de víctimas de la Red Neuronal al año 2030 (Keras)

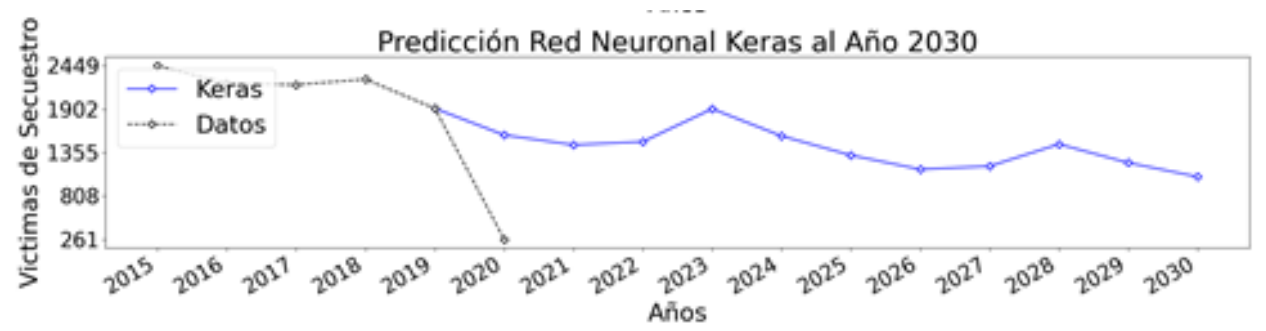

\section{Fuente: Elaboración propia}

En la Figura 9 se muestran gráficas de dispersión según las estimaciones de cada implementación. El objetivo es comparar los valores esperados del conjunto de datos con los resultados del modelo. Entre más se acerquen los puntos a la recta graficada mejor se ajusta el modelo. Se puede observar que hay valores de la máquina de soporte vectorial que no son los esperados y se encuentran muy aislados, esto puede explicar los valores obtenidos del RMSE.

\section{Figura 9. Gráficas de dispersión para cada modelo}
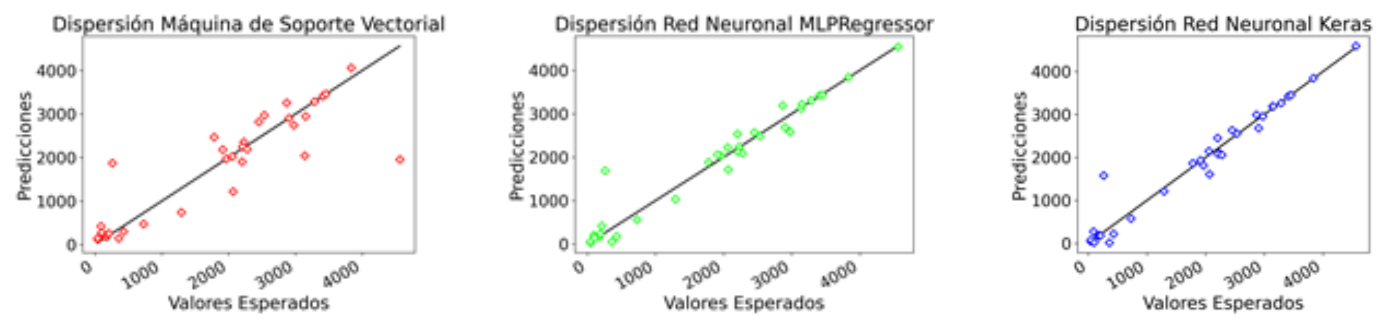

Fuente: Elaboración propia 


\section{Conclusiones}

Este documento presentó la evaluación de dos modelos: Máquinas de Soporte Vectorial para regresión (SVR) y Redes Neuronales donde se pudo estimar la cantidad de víctimas de secuestros en los próximos diez años en todo el territorio colombiano, y se encontró que la implementación de la Red Neuronal tuvo mejor comportamiento que la Máquina de Soporte Vectorial.

La cantidad o granularidad de los casos y el conteo de víctimas no está detallado por días o por meses, lo que ofrece cierta dificultad en modelar un conjunto de entrenamiento para obtener predicciones con menor RMSE, por lo que los datos actuales no son suficientes.

La evaluación de la Red Neuronal se llevó a cabo mediante un proceso de prueba y error hasta estimar la estructura presentada en este documento, sin embargo esto no garantiza la reproducción de los resultados debido a que los algoritmos de entrenamiento tienen un componente aleatorio, aun así se pueden esperar instancias entrenadas que se acerquen a los valores RMSE presentados.

Los valores de la raíz del error cuadrático medio para la Máquina de Soporte Vectorial fueron 608.98 para entrenamiento y 686.40 para pruebas, mientras que los valores de la mejor Red Neuronal son de 148.60 para entrenamiento y 555.03 para pruebas. Considerando estos valores, se concluye que los valores dados por la Red Neuronal en cualquiera de sus dos implementaciones son fiables o de referencia para los próximos diez años incluido 2020.

El modelo permite predecir que con el paso del tiempo, la cantidad de secuestros irá disminuyendo y esto se puede correlacionar con la implementación de los acuerdos de paz y otras políticas públicas. Se sugiere continuar con el trabajo, validando los valores predichos por la Red Neuronal en los próximos años con los nuevos reportes que se vayan registrando en el Portal de Datos Abiertos.

\section{Referencias}

1. J. L. G. González, «Los delitos de lesa humanidad», Revista de la Facultad de Derecho, n.o 30, pp. 153-170, 2011.

2. Secuestros, conteo Ranking, 2019 knoema.com. [En línea]. Disponible En: https://knoema.es//atlas/ topics/Estadística-criminal/Ataques-Secuestros-Robos-Violaciónes-Sexuales/Secuestros-conteo.

3. L. F. Trejos Rosero, «Colombia: Una revisión teórica de su conflicto armado», Revista Enfoques: Ciencia Política y Administración Pública, vol. 11, n.o 18, pp. 55-75, 2013.

4. ONU, Cooperación internacional para prevenir, combatir y eliminar el secuestro y prestar asistencia a las víctimas. Naciones Unidas, 2004.

5. J. D. Rodríguez-Ortega, Y. A. Duarte Velásquez, C. Gómez Toro, y J. A. Cadavid Carmona, «Seguridad ciudadana, violencia y criminalidad: una visión holística y criminológica de las cifras estadísticas del 2018», Revista Criminalidad, vol. 61, pp. 9-33, 2019.

6. D. M. Agudelo V., «El Horror de lo incierto. Intervención Psicológica con Víctimas de Secuestro», Psicología desde el Caribe, n.o 6, pp. 20-38, 2000.

7. Conteo de Víctimas, Datos Abiertos Colombia. [En línea]. Disponible En: https://www.datos.gov.co/ Justicia-y-Derecho/Conteo-de-V-ctimas/sft7-9im5. 
8. X. Meng, L. Nie, y J. Song, «Big data-based prediction of terrorist attacks», Computers \& Electrical Engineering, vol. 77, pp. 120-127, jul. 2019, DOI: 10.1016/j.compeleceng.2019.05.013.

9. H. Ordóñez, C. Cobos, y V. Bucheli, «Modelo de machine learning para la predicción de las tendencias de hurto en Colombia», 2020, vol. 29, pp. 494-506, feb. 2020.

10. D. P. Kingma y J. Ba, «Adam: A Method for Stochastic Optimization», arXiv:1412.6980 [cs], ene. 2017, Accedido: may 05, 2020. [En línea]. Disponible en: http://arxiv.org/abs/1412.6980.

11. A. Sánchez López de Mesa et al., Mapas y Factores de riesgo electoral. Elecciones de autoridades locales Colombia 2019, vol. 1. Bogota, Colombia, 2019.

12. Y. Chen, F. Li, J. Wang, B. Tang, y X. Zhou, «Quantum recurrent encoder-decoder neural network for performance trend prediction of rotating machinery», Knowledge-Based Systems, vol. 197, p. 105863, jun. 2020, DOI: 10.1016/j.knosys.2020.105863.

13. K. Pugalenthi, H. Park, y N. Raghavan, «Prognosis of power MOSFET resistance degradation trend using artificial neural network approach», Microelectronics Reliability, vol. 100-101, p. 113467, sep. 2019, DOI: 10.1016/j.microrel.2019.113467.

14. A. A. Albert, L. F. de Mingo López, y N. G. Blas, «Multilinear Weighted Regression (MWE) with Neural Networks for trend prediction», Applied Soft Computing, vol. 82, p. 105555, sep. 2019, DOI: 10.1016/j. asoc.2019.105555.

15. [A. P. Balsamà, L. De Biase, G. Janssens-Maenhout, y V. Pagliari, «Near-term projection of anthropogenic emission trends using neural networks», Atmospheric Environment, vol. 89, pp. 581-592, jun. 2014, DOI: 10.1016/j.atmosenv.2014.02.046.

16. L. Liu, M. Han, Y. Zhou, y Y. Wang, «LSTM Recurrent Neural Networks for Influenza Trends Prediction», en Bioinformatics Research and Applications, Cham, 2018, pp. 259-264, DOI: 10.1007/978-3-319-949680_25.

17. X. Zhao y J. Tang, «Exploring Transfer Learning for Crime Prediction», nov. 2017, pp. 1158-1159, doi: 10.1109/ICDMW.2017.165.

18. P. Das y A. Das, «Application of Classification Techniques for Prediction and Analysis of Crime in India», 2019, pp. 191-201.

19. S. Kim, P. Joshi, P. Kalsi, y P. Taheri, «Crime Analysis Through Machine Learning», nov. 2018, pp. 415420, DOI: 10.1109/IEMCON.2018.8614828.

20. G. Hajela, M. Chawla, y A. Rasool, «A Clustering Based Hotspot Identification Approach For Crime Prediction», Procedia Computer Science, vol. 167, pp. 1462-1470, ene. 2020, DOI: 10.1016/j. procs.2020.03.357.

21. S. Chatterjee, S. Das, S. Banerjee, y U. Biswas, «An Approach Towards Development of a Predictive Model for Female Kidnapping in India Using R Programming: eHaCON 2018, Kolkata, India», 2019, pp. 489-503.

22. J. Q. Yuki, Md. M. Q. Sakib, Z. Zamal, K. M. Habibullah, y A. K. Das, «Predicting Crime Using Time and Location Data», en Proceedings of the 2019 7th International Conference on Computer and Communications Management, Bangkok, Thailand, jul.2019, pp. 124-128, DOI: 10.1145/3348445.3348483.

23. P. Chen y J. Kurland, «Time, Place, and Modus Operandi: A Simple Apriori Algorithm Experiment for Crime Pattern Detection», jul. 2018, pp. 1-3, DOI: 10.1109/IISA.2018.8633657.

24. A. Ristea, M. Boni, B. Resch, M. Gerber, y M. Leitner, «Spatial crime distribution and prediction for sporting events using social media», International Journal of Geographical Information Science, pp. 1-32, feb. 2020, DOI: 10.1080/13658816.2020.1719495. 
25. Q. Wang, G. Jin, X. Zhao, Y. Feng, y J. Huang, «CSAN: A neural network benchmark model for crime forecasting in spatio-temporal scale», Knowledge-Based Systems, vol. 189, p. 105120, feb. 2020, DOI: 10.1016/j.knosys.2019.105120.

26. Y. Zhang y T. Cheng, «Graph deep learning model for network-based predictive hotspot mapping of sparse spatio-temporal events», Computers, Environment and Urban Systems, vol. 79, p. 101403, ene. 2020, DOI: 10.1016/j.compenvurbsys.2019.101403.

27. G. N. Kouziokas, «The application of artificial intelligence in public administration for forecasting high crime risk transportation areas in urban environment», Transportation Research Procedia, vol. 24, pp. 467-473, ene. 2017, DOI: 10.1016/j.trpro.2017.05.083.

28. S. W. Palocsay, P. Wang, y R. G. Brookshire, «Predicting criminal recidivism using neural networks», Socio-Economic Planning Sciences, vol. 34, n.o 4, pp. 271-284, dic. 2000, DOI: 10.1016/S00380121(00)00003-3. 